\title{
High-energy electroproduction in an atomic field
}

\author{
P. A. Krachkov* and A. I. Milstein $\dagger^{\dagger}$ \\ Budker Institute of Nuclear Physics, 630090 Novosibirsk, Russia
}

(Dated: July 17, 2021)

\begin{abstract}
The differential cross section of high-energy electroproduction in the electric field of heavy atoms is derived. The result is obtained with the exact account of the atomic field by means of the quasiclassical approximation to the wave functions in the external field. The Coulomb corrections substantially modify the differential cross section compared with the Born result. They lead to the azimuth asymmetry in the differential cross section for the polarized incoming electron. The Coulomb corrections to the total cross section are obtained in the leading logarithmic approximation.
\end{abstract}

PACS numbers: 12.20.Ds, 32.80.-t

Keywords: electroproduction, photoproduction, bremsstrahlung, Coulomb corrections, screening

*Electronic address: peter phys@mail.ru

${ }^{\dagger}$ Electronic address: A.I.Milstein@inp.nsk.su 


\section{INTRODUCTION}

The process of $e^{+} e^{-}$pair production at collisions of high-energy electron with atoms, which is commonly referred to as electroproduction or the trident process, is one of the most interesting and important QED processes. This process should be taken into account when considering electromagnetic showers in detectors. Electroproduction is also important in some fixed target experiments, see, e.g., dark-photon search experiments [1, 2]. In these experiments electroproduction is the basic irreducible background process.

The process of electroproduction has been under consideration for a long time. The earliest papers are those of Bhabha [3, 4] and Racah [5, 6]. In Refs. [3, 4] calculations were performed with the use of the Weizsäcker-Williams approximation (see, e.g., Ref. [7]), which allows one to calculate the total cross section of the process in the leading approximation with respect to the parameter $\ln (\varepsilon / m)$, here $m$ is the electron mass and $\varepsilon$ is the energy of the incoming electron, $\hbar=c=1$. In Refs. [5, 6] the total cross section was obtained without restrictions needed for applicability of the Weizsäcker-Williams approximation. However the effect of Fermi statistics for two outgoing electrons were not taken into account at that time. In Ref. [8] the approximate result for the total cross section, which is in good agreement with that given by Racah [5, 6], was obtained. In Refs. [9, 10] it is shown that the Bhabha's formula for the total electroproduction cross section has a good accuracy at $\varepsilon \gtrsim 10 \mathrm{GeV}$. The first numerical evaluation of the electroproduction cross section was performed in Ref. [11], where the differential cross section of high-energy electroproduction was obtained taking into account the effects of Fermi statistics. The differential cross section of high-energy electroproduction for massless leptons was derived in [12-15].

In all papers mentioned above the cross sections were obtained in the leading in the parameter $\eta=Z \alpha$ approximation (in the Born approximation), where $Z$ is the atomic charge number and $\alpha$ is the fine-structure constant. The Coulomb corrections for the differential cross section of electroproduction (the difference between the exact in $\eta$ result and the Born result) have not been derived till now. However, it is well known from the results for the differential cross sections of photoproduction and bremsstrahlung that the Coulomb corrections

may drastically change the result for heavy atoms [16-18]. It is very difficult to calculate the Coulomb corrections to the electroproduction cross section because the amplitude of this process contains four wave functions in the atomic field, see Fig. 1 where the corresponding 

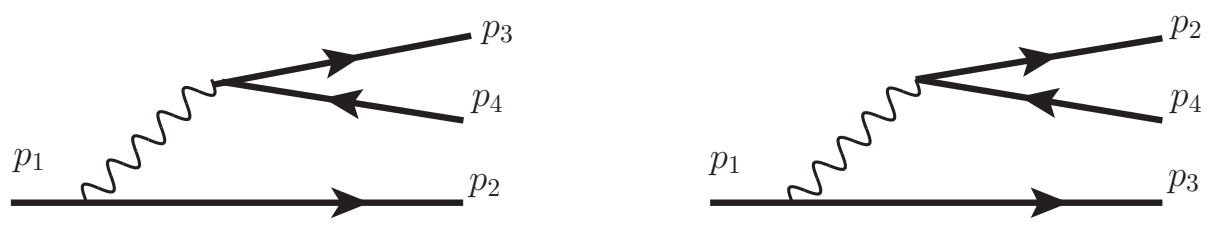

FIG. 1: Diagrams for the amplitude of the process $e^{-} Z \rightarrow e^{-} e^{+} e^{-} Z$. Wavy line denotes the photon propagator, straight lines denote the wave functions in the atomic field.

Feynman diagrams in the Furry representation are shown. Even in the case of photoproduction and bremsstrahlung, where the corresponding amplitudes contain only two wave functions in the atomic field, exact calculations of the Coulomb corrections for any energies are very complicated task. Fortunately, the use of the quasiclassical approximation for the electron wave and Green's functions in the atomic field significantly simplifies calculations at high electron energies (though does not make them simple).

At high energies and small angles between outgoing and incoming particles, the main contribution to the processes in the atomic field is given by large angular momenta of the particles. The quasiclassical approximation provides a possibility to account for the contribution of this large angular-momenta region. For the Coulomb potential, the wave functions in the leading quasiclassical approximation are the famous Furry-SommerfeldMaue wave functions [19, 20] (see also Ref. [7]). For the atomic potential, the wave functions and the Green's functions in the leading and next-to-leading quasiclassical approximation have been derived in Refs. [21, 22]. Using the quasiclassical approximation the exact in $\eta$ differential cross sections for photoproduction and bremsstrahlung in the atomic field have been obtained in Refs. [16 18, 23] in the leading quasiclassical approximation. In Refs. [22] and [24] the differential cross sections for bremsstrahlung and photoproduction have been obtained exactly in $\eta$ with the first quasiclassical corrections taken into account. In Refs. [25] and [26] the cross section of $e^{+} e^{-}$photoproduction accompanied by bremsstrahlung and the cross section of double bremsstrahlung have been obtained exactly in $\eta$ in the leading quasiclassical approximation.

In the present paper, we apply the quasiclassical approach to investigate, exactly in $\eta$, the differential cross section of high-energy electroproduction. The Coulomb corrections to the cross section of photoproduction are determined by the region of small impact parameters 
$\rho \sim \lambda_{C}=1 / m$, while the Coulomb corrections to the cross section of bremsstrahlung are determined by large impact parameters $\rho \sim \min \left\{\lambda_{C} \varepsilon \varepsilon^{\prime} /(m \omega), r_{s c r}\right\}$, where $\omega$ is the energy of emitted photon, $\varepsilon^{\prime}=\varepsilon-\omega$, and $r_{s c r} \sim \lambda_{C} Z^{-1 / 3} / \alpha$ is the screening radius. For the differential cross section of electroproduction, both regions of small and large impact parameters give the contribution to the Coulomb corrections. We show that the Coulomb corrections for heavy atoms drastically change the result compared with that obtained in the Born approximation.

\section{GENERAL DISCUSSION}

The differential cross section of high-energy electroproduction in an atomic field reads [7]

$$
d \sigma=\frac{\alpha^{2}}{(2 \pi)^{8}} \varepsilon_{2}^{2} \varepsilon_{3}^{2} \varepsilon_{4}^{2} d \varepsilon_{3} d \varepsilon_{4} d \Omega_{2} d \Omega_{3} d \Omega_{4}|\mathcal{T}|^{2},
$$

where $d \Omega_{2}, d \Omega_{3}$ are the solid angles corresponding to the momenta $\boldsymbol{p}_{2}$ and $\boldsymbol{p}_{3}$ of the outgoing electrons, $d \Omega_{4}$ is the solid angle corresponding to the positron momentum $\boldsymbol{p}_{4}, \boldsymbol{p}_{1}$ is the incoming electron momentum (see Fig.1), $\varepsilon_{1}=\varepsilon_{2}+\varepsilon_{3}+\varepsilon_{4}$ is the incoming electron energy, and $\varepsilon_{i}=\sqrt{p_{i}^{2}+m^{2}}$. Below we assume that $\varepsilon_{i} \gg m$. The matrix element $\mathcal{T}$ reads

$$
\begin{aligned}
\mathcal{T} & =T+\widetilde{T}, \quad \widetilde{T}=-T(2 \leftrightarrow 3), \\
T & =\sum_{a, b=1}^{3} \int \frac{d \boldsymbol{k}}{(2 \pi)^{3}} \mathcal{D}^{a b} j^{a} J^{b}, \quad \mathcal{D}^{a b}=-\frac{4 \pi}{\omega^{2}-k^{2}+i 0}\left(\delta^{a b}-\frac{k^{a} k^{b}}{\omega^{2}}\right), \\
\boldsymbol{j} & =\int d \boldsymbol{r} e^{-i \boldsymbol{k} \cdot \boldsymbol{r}} \bar{u}_{\boldsymbol{p}_{2}}^{(-)}(\boldsymbol{r}) \boldsymbol{\gamma} u_{\boldsymbol{p}_{1}}^{(+)}(\boldsymbol{r}), \quad \boldsymbol{J}=\int d \boldsymbol{r} e^{i \boldsymbol{k} \cdot \boldsymbol{r}} \bar{u}_{\boldsymbol{p}_{3}}^{(-)}(\boldsymbol{r}) \boldsymbol{\gamma} v_{\boldsymbol{p}_{4}}^{(+)}(\boldsymbol{r}), \\
\omega & =\varepsilon_{1}-\varepsilon_{2}=\varepsilon_{3}+\varepsilon_{4},
\end{aligned}
$$

where $D^{\mu \nu}$ is a photon propagator $\left(D^{\mu 0}=0\right), \gamma^{\nu}$ are the Dirac matrices, $u_{p}^{(+)}(\boldsymbol{r})$ and $u_{p}^{(-)}(\boldsymbol{r})$ are the positive-energy solutions of the Dirac equation in the atomic potential $V(r), v_{\boldsymbol{p}}^{(+)}(\boldsymbol{r})$ is the negative-energy solution of the Dirac equation in the atomic potential, the superscripts $(-)$ and $(+)$ indicate that the asymptotic forms of the wave functions contain at large distances $r$, in addition to the plane wave, the spherical convergent and divergent waves, respectively. We calculate the matrix element of electroproduction in the leading quasiclassical approximation. In this case the wave functions have the form [21]

$$
\begin{aligned}
& \bar{u}_{\boldsymbol{p}}^{(-)}(\boldsymbol{r})=\bar{u}_{p}\left[f_{0}(\boldsymbol{r}, \boldsymbol{p})-\boldsymbol{\alpha} \cdot \boldsymbol{f}_{1}(\boldsymbol{r}, \boldsymbol{p})\right], \\
& u_{\boldsymbol{p}}^{(+)}(\boldsymbol{r})=\left[g_{0}(\boldsymbol{r}, \boldsymbol{p})-\boldsymbol{\alpha} \cdot \boldsymbol{g}_{1}(\boldsymbol{r}, \boldsymbol{p})\right] u_{\boldsymbol{p}},
\end{aligned}
$$




$$
\begin{aligned}
& v_{\boldsymbol{p}}^{(+)}(\boldsymbol{r})=\left[G_{0}(\boldsymbol{r}, \boldsymbol{p})+\boldsymbol{\alpha} \cdot \boldsymbol{G}_{1}(\boldsymbol{r}, \boldsymbol{p})\right] v_{\boldsymbol{p}} \\
& u_{\boldsymbol{p}}=\sqrt{\frac{\varepsilon_{p}+m}{2 \varepsilon_{p}}}\left(\begin{array}{c}
\phi \\
\frac{\boldsymbol{\sigma} \cdot \boldsymbol{p}}{\varepsilon_{p}+m} \phi
\end{array}\right), \quad v_{\boldsymbol{p}}=\sqrt{\frac{\varepsilon_{p}+m}{2 \varepsilon_{p}}}\left(\begin{array}{c}
\frac{\boldsymbol{\sigma} \cdot \boldsymbol{p}}{\varepsilon_{p}+m} \chi \\
\chi
\end{array}\right),
\end{aligned}
$$

where $\phi$ and $\chi$ are spinors, $\boldsymbol{\alpha}=\gamma^{0} \boldsymbol{\gamma}$, and $\boldsymbol{\sigma}$ are the Pauli matrices. The functions $f_{0}$ and $f_{1}$ read

$$
\begin{aligned}
& f_{0}(\boldsymbol{r}, \boldsymbol{p})=-\frac{i}{\pi} e^{-i \boldsymbol{p} \cdot \boldsymbol{r}} \int d \boldsymbol{Q} \exp \left[i Q^{2}-i \int_{0}^{\infty} d x V\left(\boldsymbol{r}_{p}\right)\right], \\
& \boldsymbol{f}_{1}(\boldsymbol{r}, \boldsymbol{p})=\frac{1}{2 \varepsilon_{p}}(i \boldsymbol{\nabla}-\boldsymbol{p}) f_{0}(\boldsymbol{r}, \boldsymbol{p}), \\
& \boldsymbol{r}_{p}=\boldsymbol{r}+x \boldsymbol{n}_{p}+\sqrt{\frac{2 x}{\varepsilon_{p}}} \boldsymbol{Q},
\end{aligned}
$$

where $\boldsymbol{Q}$ is a two-dimensional vector perpendicular to the vector $\boldsymbol{n}_{p}=\boldsymbol{p} / p$. The expressions for the functions $g_{0}$ and $\boldsymbol{g}_{1}$ follow from the relations

$$
g_{0}(\boldsymbol{r}, \boldsymbol{p})=f_{0}(\boldsymbol{r},-\boldsymbol{p}), \quad \boldsymbol{g}_{1}(\boldsymbol{r}, \boldsymbol{p})=f_{1}(\boldsymbol{r},-\boldsymbol{p}),
$$

and the expressions for the functions $G_{0}$ and $\boldsymbol{G}_{1}$ can be obtained from the functions $f_{0}$ and $\boldsymbol{f}_{1}$, respectively, by the replacement $V\left(\boldsymbol{r}_{p}\right) \rightarrow-V\left(\boldsymbol{r}_{p}\right)$.

It is convenient to calculate the matrix element for definite helicities of the particles. Let $\mu_{i}$ be a sign of the helicity of a particle with the momentum $\boldsymbol{p}_{i}$. We direct the $z$-axis along a unit vector $\boldsymbol{\nu}$ assuming that the angles between $\boldsymbol{\nu}$ and $\boldsymbol{p}_{i}$ are small. The final result will be independent of the direction of $\boldsymbol{\nu}$. Then, to calculate the matrix element, we use the matrices $\mathcal{F}=u_{\boldsymbol{p}_{1} \mu_{1}} \bar{u}_{\boldsymbol{p}_{2} \mu_{2}}$ and $\widetilde{\mathcal{F}}=v_{\boldsymbol{p}_{4} \mu_{4}} \bar{u}_{\boldsymbol{p}_{3} \mu_{3}}[22,25]$

$$
\begin{aligned}
& \mathcal{F}=\frac{1}{8}\left(a_{\mu_{1} \mu_{2}}+\Sigma \cdot \boldsymbol{b}_{\mu_{1} \mu_{2}}\right)\left[\gamma^{0}\left(1+P_{1} P_{2}\right)+\gamma^{0} \gamma^{5}\left(P_{1}+P_{2}\right)+\left(1-P_{1} P_{2}\right)-\gamma^{5}\left(P_{1}-P_{2}\right)\right], \\
& \widetilde{\mathcal{F}}=\frac{1}{8}\left(\widetilde{a}_{\mu_{3} \mu_{4}}+\Sigma \cdot \widetilde{\boldsymbol{b}}_{\mu_{3} \mu_{4}}\right)\left[\gamma^{0}\left(P_{3}-P_{4}\right)+\gamma^{0} \gamma^{5}\left(1-P_{3} P_{4}\right)-\left(P_{3}+P_{4}\right)-\gamma^{5}\left(1+P_{3} P_{4}\right)\right] .
\end{aligned}
$$

Here $P_{i}=\mu_{i} p_{i} /\left(\varepsilon_{i}+m\right), \boldsymbol{\Sigma}=-\gamma^{5} \boldsymbol{\alpha}, \gamma^{5}=-i \gamma^{0} \gamma^{1} \gamma^{2} \gamma^{3} ; a_{\mu_{1} \mu_{2}}, \boldsymbol{b}_{\mu_{1} \mu_{2}}, \widetilde{a}_{\mu_{3} \mu_{4}}$, and $\widetilde{\boldsymbol{b}}_{\mu_{3} \mu_{4}}$ are

$$
\begin{aligned}
& a_{\mu \mu}=1, \quad a_{\mu \bar{\mu}}=\frac{\mu}{\sqrt{2}} \boldsymbol{s}_{\mu} \cdot \boldsymbol{\theta}_{12}, \\
& \boldsymbol{b}_{\mu \mu}=\mu \boldsymbol{\nu}+\frac{\mu}{2}\left(\boldsymbol{\theta}_{1}+\boldsymbol{\theta}_{2}\right)+\frac{i}{2}\left[\boldsymbol{\theta}_{12} \times \boldsymbol{\nu}\right], \\
& \boldsymbol{b}_{\mu \bar{\mu}}=\sqrt{2} \boldsymbol{s}_{\mu}-\frac{1}{\sqrt{2}}\left(\boldsymbol{s}_{\mu}, \boldsymbol{\theta}_{1}+\boldsymbol{\theta}_{2}\right) \boldsymbol{\nu},
\end{aligned}
$$




$$
\begin{aligned}
& \widetilde{a}_{\mu \bar{\mu}}=\mu, \quad \widetilde{a}_{\mu \mu}=-\frac{1}{\sqrt{2}} \boldsymbol{s}_{\mu}^{*} \cdot \boldsymbol{\theta}_{34}, \\
& \widetilde{\boldsymbol{b}}_{\mu \bar{\mu}}=\boldsymbol{\nu}+\frac{1}{2}\left(\boldsymbol{\theta}_{3}+\boldsymbol{\theta}_{4}\right)-\frac{i \mu}{2}\left[\boldsymbol{\theta}_{34} \times \boldsymbol{\nu}\right] \\
& \widetilde{\boldsymbol{b}}_{\mu \mu}=-\mu \sqrt{2} \boldsymbol{s}_{\mu}^{*}+\frac{\mu}{\sqrt{2}}\left(\boldsymbol{s}_{\mu}^{*}, \boldsymbol{\theta}_{3}+\boldsymbol{\theta}_{4}\right) \boldsymbol{\nu} \\
& \boldsymbol{s}_{\mu}=\frac{1}{\sqrt{2}}\left(\boldsymbol{e}_{x}+i \mu \boldsymbol{e}_{y}\right)
\end{aligned}
$$

where $\bar{\mu}=-\mu, \boldsymbol{e}_{x}$ and $\boldsymbol{e}_{y}$ are two orthogonal unit vectors perpendicular to $\boldsymbol{\nu}, \boldsymbol{\theta}_{i}=\boldsymbol{p}_{i \perp} / p_{i}$, $\boldsymbol{\theta}_{i j}=\boldsymbol{\theta}_{i}-\boldsymbol{\theta}_{j}$, and the notation $\boldsymbol{X}_{\perp}=\boldsymbol{X}-(\boldsymbol{X} \cdot \boldsymbol{\nu}) \boldsymbol{\nu}$ for any vector $\boldsymbol{X}$ is used.

It is convenient to write the photon propagator $\mathcal{D}^{a b}$ as follows:

$$
\begin{aligned}
& \mathcal{D}^{a b}=\mathcal{D}_{\perp}^{a b}+\mathcal{D}_{\|}^{a b}, \\
& \mathcal{D}_{\perp}^{a b}=-\frac{4 \pi}{\omega^{2}-k^{2}+i 0}\left(\delta^{a b}-\frac{k^{a} k^{b}}{k^{2}}\right)=-\frac{4 \pi}{\omega^{2}-k^{2}+i 0} \sum_{\lambda= \pm} s_{\lambda}^{a *} s_{\lambda}^{b}, \\
& \mathcal{D}_{\|}^{a b}=-\frac{4 \pi}{\omega^{2} k^{2}} k^{a} k^{b}=-\frac{4 \pi}{\omega^{2}} \nu^{a} \nu^{b},
\end{aligned}
$$

where we direct the vector $\boldsymbol{\nu}$ along the vector $\boldsymbol{k}$. Substituting this expression to Eq.(2) we obtain for $T$

$$
\begin{aligned}
& T=T_{\perp}+T_{\|}, \\
& T_{\perp}=-4 \pi \sum_{\lambda= \pm} \int \frac{d \boldsymbol{k} j_{\lambda} J_{\lambda}}{(2 \pi)^{3}\left(\omega^{2}-k^{2}+i 0\right)}, \\
& T_{\|}=-\frac{4 \pi}{\omega^{2}} \int \frac{d \boldsymbol{k}}{(2 \pi)^{3}} j_{\|} J_{\|}, \\
& j_{\lambda}=\boldsymbol{j} \cdot \boldsymbol{s}_{\lambda}^{*}, \quad J_{\lambda}=\boldsymbol{J} \cdot \boldsymbol{s}_{\lambda}, \quad j_{\|}=\boldsymbol{j} \cdot \boldsymbol{\nu}, \quad J_{\|}=\boldsymbol{J} \cdot \boldsymbol{\nu} .
\end{aligned}
$$

The functions $\boldsymbol{j}$ and $\boldsymbol{J}$ correspond to the matrix elements of virtual photon bremsstrahlung and pair production by virtual photon, respectively. The calculation of these functions can be performed in the same way as it was done for the real bremsstrahlung cross section [22, 23] and for the pair production cross section by a real photon [25]. As a result we obtain for the matrix elements $j_{\lambda}$ and $j_{\|}$of virtual bremsstrahlung

$$
\begin{aligned}
& j_{\lambda}=-A(\boldsymbol{\Delta})\left[\delta_{\mu_{1} \mu_{2}}\left(\varepsilon_{1} \delta_{\lambda \mu_{1}}+\varepsilon_{2} \delta_{\lambda \bar{\mu}_{1}}\right)\left(\boldsymbol{s}_{\lambda}^{*}, \frac{\boldsymbol{\theta}_{2}}{\varepsilon_{1} D_{1}}+\frac{\boldsymbol{\theta}_{1}}{\varepsilon_{2} D_{2}}\right)\right. \\
& \left.+\delta_{\mu_{1} \bar{\mu}_{2}} \delta_{\lambda \mu_{1}} \frac{m \omega \mu_{1}}{\sqrt{2} \varepsilon_{1} \varepsilon_{2}}\left(\frac{1}{D_{1}}+\frac{1}{D_{2}}\right)\right],
\end{aligned}
$$




$$
\begin{aligned}
& j_{\|}=-A(\boldsymbol{\Delta}) \delta_{\mu_{1} \mu_{2}}\left(\frac{1}{D_{1}}+\frac{1}{D_{2}}\right), \\
& A(\boldsymbol{\Delta})=-\frac{i}{\Delta_{\perp}^{2}} \int d \boldsymbol{r} \exp [-i \boldsymbol{\Delta} \cdot \boldsymbol{r}-i \chi(\rho)] \boldsymbol{\Delta}_{\perp} \cdot \nabla_{\perp} V(r), \\
& \chi(\rho)=\int_{-\infty}^{\infty} d z V\left(\sqrt{z^{2}+\rho^{2}}\right), \\
& D_{1}=\frac{\Delta_{\perp}^{2}}{2 \varepsilon_{1}}+\boldsymbol{n}_{1} \cdot \boldsymbol{\Delta}-i 0, \quad D_{2}=\frac{\Delta_{\perp}^{2}}{2 \varepsilon_{2}}-\boldsymbol{n}_{2} \cdot \boldsymbol{\Delta}-i 0, \\
& \boldsymbol{\Delta}=\boldsymbol{k}+\boldsymbol{p}_{2}-\boldsymbol{p}_{1}, \quad \boldsymbol{n}_{i}=\boldsymbol{p}_{i} / p_{i} .
\end{aligned}
$$

At $\Delta_{\perp} \gg \max \left(\Delta_{\|}, r_{s c r}^{-1}\right)$, where $\Delta_{\|}=\boldsymbol{\Delta} \cdot \boldsymbol{\nu}$ and $r_{s c r}$ is a screening radius, the function $A(\boldsymbol{\Delta})$ is independent of the potential shape (see Ref. [23]). It has the following asymptotic form

$$
A_{a s}(\Delta)=-\frac{4 \pi \eta\left(L \Delta_{\perp}\right)^{2 i \eta} \Gamma(1-i \eta)}{\Delta_{\perp}^{2} \Gamma(1+i \eta)}
$$

where $\Gamma(x)$ is the Euler $\Gamma$ function, a specific value of $L \sim \max \left(\Delta_{\|}, r_{s c r}^{-1}\right)$ is irrelevant because the factor $L^{2 i \eta}$ disappears in $\left|T_{\text {tot }}\right|^{2}$. At $\Delta_{\perp} \lesssim \max \left(\Delta_{\|}, r_{\text {scr }}^{-1}\right)$, the function $A(\Delta)$ strongly depends on $\Delta_{\|}$and the shape of the atomic potential [23].

The matrix elements $J_{\lambda}$ and $J_{\|}$of pair production by virtual photon read

$$
\begin{aligned}
& J_{\lambda}=J_{\lambda}^{(0)}+J_{\lambda}^{(1)}, \quad J_{\|}=J_{\|}^{(0)}+J_{\|}^{(1)}, \\
& J_{\lambda}^{(0)}=(2 \pi)^{3} \delta\left(\boldsymbol{p}_{3}+\boldsymbol{p}_{4}-\boldsymbol{k}\right)\left[\delta_{\mu_{3} \bar{\mu}_{4}}\left(\boldsymbol{s}_{\lambda}, \delta_{\lambda \mu_{3}} \boldsymbol{\theta}_{4}+\delta_{\lambda \mu_{4}} \boldsymbol{\theta}_{3}\right)-\delta_{\mu_{3} \mu_{4}} \delta_{\lambda \mu_{3}} \frac{m \omega \mu_{3}}{\sqrt{2} \varepsilon_{3} \varepsilon_{4}}\right], \\
& J_{\lambda}^{(1)}=\frac{i \varepsilon_{3} \varepsilon_{4}}{2 \pi \omega} \int_{0}^{\infty} \frac{d z}{z} e^{i z\left(p_{3}+p_{4}-k+i 0\right)} \iint d^{2} Q_{3} d^{2} Q_{4} \mathcal{J} e^{i \Phi} \\
& \times\left[\frac{\delta_{\mu_{3} \bar{\mu}_{4}}}{\omega z}\left(\boldsymbol{s}_{\lambda} \cdot \boldsymbol{Q}_{34}\right)\left(\varepsilon_{3} \delta_{\lambda \mu_{3}}-\varepsilon_{4} \delta_{\lambda \mu_{4}}\right)+\delta_{\mu_{3} \mu_{4}} \delta_{\lambda \mu_{3}} \frac{m \omega \mu_{3}}{\sqrt{2} \varepsilon_{3} \varepsilon_{4}}\right], \\
& J_{\|}^{(0)}=(2 \pi)^{3} \delta\left(\boldsymbol{p}_{3}+\boldsymbol{p}_{4}-\boldsymbol{k}\right) \delta_{\mu_{3} \bar{\mu}_{4}}, \\
& J_{\|}^{(1)}=-\frac{i \varepsilon_{3} \varepsilon_{4}}{2 \pi \omega} \int_{0}^{\infty} \frac{d z}{z} e^{i z\left(p_{3}+p_{4}-k+i 0\right)} \iint d^{2} Q_{3} d^{2} Q_{4} \mathcal{J} e^{i \Phi} \delta_{\mu_{3} \bar{\mu}_{4}}, \\
& \mathcal{J}=e^{i\left[\chi\left(Q_{4}\right)-\chi\left(Q_{3}\right)\right]}-1, \quad \Phi=\frac{\varepsilon_{3} \varepsilon_{4}}{2 \omega z} Q_{34}^{2}-\left(\varepsilon_{3} \boldsymbol{Q}_{3} \cdot \boldsymbol{\theta}_{3}+\varepsilon_{4} \boldsymbol{Q}_{4} \cdot \boldsymbol{\theta}_{4}\right),
\end{aligned}
$$

where $\boldsymbol{Q}_{34}=\boldsymbol{Q}_{3}-\boldsymbol{Q}_{4}$. The matrix elements $J_{\lambda}^{(0)}$ and $J_{\|}^{(0)}$ correspond to the virtual photon decay into $e^{+} e^{-}$pair noninteracting with the atomic field, while the matrix elements $J_{\lambda}^{(1)}$ and $J_{\|}^{(1)}$ correspond to production of pair interacting with the atomic field. Then we substitute Eqs. (10) and (12) in Eq. (2) and write the amplitudes $T_{\perp}$ and $T_{\|}$as follows

$$
T_{\perp}=T_{\perp}^{(0)}+T_{\perp}^{(1)}, \quad T_{\|}=T_{\|}^{(0)}+T_{\|}^{(1)} .
$$


Integrating over $\boldsymbol{k}$, we obtain for the terms $T_{\perp}^{(0)}$ and $T_{\|}^{(0)}$ :

$$
\begin{aligned}
& T_{\perp}^{(0)}=\frac{8 \pi \varepsilon_{3} \varepsilon_{4} A\left(\boldsymbol{\Delta}_{0}\right)}{m^{2} \omega^{2}+\varepsilon_{3}^{2} \varepsilon_{4}^{2} \theta_{34}^{2}}\left\{\delta _ { \mu _ { 1 } \mu _ { 2 } } \delta _ { \mu _ { 3 } \overline { \mu } _ { 4 } } \left[\frac{\varepsilon_{3}}{\omega^{2}}\left(\boldsymbol{s}_{\mu_{3}}^{*} \cdot \boldsymbol{X}\right)\left(\boldsymbol{s}_{\mu_{3}} \cdot \boldsymbol{\theta}_{34}\right)\left(\varepsilon_{1} \delta_{\mu_{1} \mu_{3}}+\varepsilon_{2} \delta_{\mu_{1} \mu_{4}}\right)\right.\right. \\
& \left.-\frac{\varepsilon_{4}}{\omega^{2}}\left(\boldsymbol{s}_{\mu_{4}}^{*} \cdot \boldsymbol{X}\right)\left(\boldsymbol{s}_{\mu_{4}} \cdot \boldsymbol{\theta}_{34}\right)\left(\varepsilon_{1} \delta_{\mu_{1} \mu_{4}}+\varepsilon_{2} \delta_{\mu_{1} \mu_{3}}\right)\right] \\
& -\frac{m \mu_{1}}{\sqrt{2} \varepsilon_{1} \varepsilon_{2}} R \delta_{\mu_{1} \bar{\mu}_{2}} \delta_{\mu_{3} \bar{\mu}_{4}}\left(\boldsymbol{s}_{\mu_{1}} \cdot \boldsymbol{\theta}_{34}\right)\left(-\varepsilon_{3} \delta_{\mu_{1} \mu_{3}}+\varepsilon_{4} \delta_{\mu_{1} \mu_{4}}\right) \\
& \left.+\frac{m \mu_{3}}{\sqrt{2} \varepsilon_{3} \varepsilon_{4}} \delta_{\mu_{1} \mu_{2}} \delta_{\mu_{3} \mu_{4}}\left(\boldsymbol{s}_{\mu_{3}}^{*} \cdot \boldsymbol{X}\right)\left(\varepsilon_{1} \delta_{\mu_{3} \mu_{1}}+\varepsilon_{2} \delta_{\mu_{3} \bar{\mu}_{1}}\right)+\frac{m^{2} \omega^{2}}{2 \varepsilon_{1} \varepsilon_{2} \varepsilon_{3} \varepsilon_{4}} R \delta_{\mu_{1} \bar{\mu}_{2}} \delta_{\mu_{3} \mu_{4}} \delta_{\mu_{1} \mu_{3}}\right\}, \\
& T_{\|}^{(0)}=-\frac{8 \pi}{\omega^{2}} A\left(\boldsymbol{\Delta}_{0}\right) R \delta_{\mu_{1} \mu_{2}} \delta_{\mu_{3} \bar{\mu}_{4}} .
\end{aligned}
$$

Here

$$
\begin{aligned}
& \boldsymbol{\Delta}_{0}=\boldsymbol{p}_{2}+\boldsymbol{p}_{3}+\boldsymbol{p}_{4}-\boldsymbol{p}_{1}, \quad \boldsymbol{\Delta}_{0 \perp}=\varepsilon_{2} \boldsymbol{\theta}_{21}+\varepsilon_{3} \boldsymbol{\theta}_{31}+\varepsilon_{4} \boldsymbol{\theta}_{41}, \\
& \Delta_{0 \|}=-\frac{1}{2}\left[m^{2} \omega\left(\frac{1}{\varepsilon_{1} \varepsilon_{2}}+\frac{1}{\varepsilon_{3} \varepsilon_{4}}\right)+\varepsilon_{2} \theta_{21}^{2}+\varepsilon_{3} \theta_{31}^{2}+\varepsilon_{4} \theta_{41}^{2}\right] \\
& R=\frac{1}{d_{1} d_{2}}\left[\Delta_{0 \perp}^{2}\left(\varepsilon_{1}+\varepsilon_{2}\right)+2 \varepsilon_{1} \varepsilon_{2}\left(\boldsymbol{\theta}_{12} \cdot \boldsymbol{\Delta}_{0 \perp}\right)\right] \\
& \boldsymbol{X}=\frac{1}{d_{1}}\left(\varepsilon_{3} \boldsymbol{\theta}_{23}+\varepsilon_{4} \boldsymbol{\theta}_{24}\right)-\frac{1}{d_{2}}\left(\varepsilon_{3} \boldsymbol{\theta}_{13}+\varepsilon_{4} \boldsymbol{\theta}_{14}\right), \\
& d_{1}=m^{2} \omega \varepsilon_{1}\left(\frac{1}{\varepsilon_{1} \varepsilon_{2}}+\frac{1}{\varepsilon_{3} \varepsilon_{4}}\right)+\varepsilon_{2} \varepsilon_{3} \theta_{23}^{2}+\varepsilon_{2} \varepsilon_{4} \theta_{24}^{2}+\varepsilon_{3} \varepsilon_{4} \theta_{34}^{2}, \\
& d_{2}=m^{2} \omega \varepsilon_{2}\left(\frac{1}{\varepsilon_{1} \varepsilon_{2}}+\frac{1}{\varepsilon_{3} \varepsilon_{4}}\right)+\varepsilon_{2} \varepsilon_{3} \theta_{31}^{2}+\varepsilon_{2} \varepsilon_{4} \theta_{41}^{2}+\left(\varepsilon_{3} \boldsymbol{\theta}_{31}+\varepsilon_{4} \boldsymbol{\theta}_{41}\right)^{2} .
\end{aligned}
$$

These amplitudes correspond to production of $e^{+} e^{-}$pair non-interacting with the atomic field, so that they have the dependence on the atomic potential similar to that of the bremsstrahlung amplitude, see, e.g., [23, 26].

To derive the terms $T_{\perp}^{(1)}$ and $T_{\|}^{(1)}$, we take the integral over $k_{z}$ by closing the contour of integration in the lower half-plane of the complex variable $k_{z}$. Then the main contribution to the integral is given by the pole of the function $1 / D_{2}$ in Eq. (10). We have

$$
\begin{aligned}
& T_{\perp}^{(1)}=-\frac{\varepsilon_{1} \varepsilon_{3} \varepsilon_{4}}{2 \pi^{2} \omega} \int \frac{d \boldsymbol{\Delta}_{\perp} A\left(\boldsymbol{\Delta}_{\perp}\right)}{m^{2} \omega^{2}+\varepsilon_{1}^{2} Y^{2}} \int_{0}^{\infty} \frac{d z}{z} \exp \left(-\frac{i z}{2} \Phi_{1}\right) \iint d^{2} Q_{3} d^{2} Q_{4} \mathcal{J} e^{i \Phi_{2}} \\
& \times\left\{\frac { \delta _ { \mu _ { 1 } \mu _ { 2 } } \delta _ { \mu _ { 3 } \overline { \mu } _ { 4 } } } { \omega ^ { 2 } z } \left[\varepsilon_{1}\left(\varepsilon_{3} \delta_{\mu_{1} \mu_{3}}-\varepsilon_{4} \delta_{\mu_{1} \mu_{4}}\right)\left(\boldsymbol{s}_{\mu_{1}}^{*} \cdot \boldsymbol{Y}\right)\left(\boldsymbol{s}_{\mu_{1}} \cdot \boldsymbol{Q}_{34}\right)\right.\right. \\
& \left.+\varepsilon_{2}\left(\varepsilon_{3} \delta_{\mu_{1} \bar{\mu}_{3}}-\varepsilon_{4} \delta_{\mu_{1} \bar{\mu}_{4}}\right)\left(\boldsymbol{s}_{\mu_{1}} \cdot \boldsymbol{Y}\right)\left(\boldsymbol{s}_{\mu_{1}}^{*} \cdot \boldsymbol{Q}_{34}\right)\right]-\delta_{\mu_{1} \bar{\mu}_{2}} \delta_{\mu_{3} \bar{\mu}_{4}} \frac{m \mu_{1}}{\sqrt{2} \varepsilon_{1} z}\left(\varepsilon_{3} \delta_{\mu_{1} \mu_{3}}-\varepsilon_{4} \delta_{\mu_{1} \mu_{4}}\right)\left(\boldsymbol{s}_{\mu_{1}} \cdot \boldsymbol{Q}_{34}\right) \\
& \left.+\delta_{\mu_{1} \mu_{2}} \delta_{\mu_{3} \mu_{4}} \frac{m \mu_{3}}{\sqrt{2} \varepsilon_{3} \varepsilon_{4}}\left(\varepsilon_{1} \delta_{\mu_{1} \mu_{3}}+\varepsilon_{2} \delta_{\mu_{1} \bar{\mu}_{3}}\right)\left(\boldsymbol{s}_{\mu_{3}}^{*} \cdot \boldsymbol{Y}\right)-\frac{m^{2} \omega^{2}}{2 \varepsilon_{1} \varepsilon_{3} \varepsilon_{4}} \delta_{\mu_{1} \bar{\mu}_{2}} \delta_{\mu_{3} \mu_{4}} \delta_{\mu_{1} \mu_{3}}\right\},
\end{aligned}
$$




$$
\begin{aligned}
& T_{\|}^{(1)}=\frac{\varepsilon_{3} \varepsilon_{4}}{2 \pi^{2} \omega^{3}} \int d \boldsymbol{\Delta}_{\perp} A\left(\boldsymbol{\Delta}_{\perp}\right) \int_{0}^{\infty} \frac{d z}{z} \exp \left(-\frac{i z}{2} \Phi_{1}\right) \iint d^{2} Q_{3} d^{2} Q_{4} \mathcal{J} e^{i \Phi_{2}} \delta_{\mu_{1} \mu_{2}} \delta_{\mu_{3} \bar{\mu}_{4}}, \\
& \Phi_{1}=m^{2} \omega\left(\frac{1}{\varepsilon_{1} \varepsilon_{2}}+\frac{1}{\varepsilon_{3} \varepsilon_{4}}\right)+\frac{\varepsilon_{1}}{\varepsilon_{2} \omega} Y^{2}, \quad \boldsymbol{Y}=\boldsymbol{\Delta}_{\perp}-\varepsilon_{2} \boldsymbol{\theta}_{21}, \\
& \Phi_{2}=\frac{\varepsilon_{3} \varepsilon_{4}}{2 \omega z} Q_{34}^{2}+\frac{\varepsilon_{3}}{\omega} \boldsymbol{Q}_{3} \cdot\left(\boldsymbol{Y}-\omega \boldsymbol{\theta}_{31}\right)+\frac{\varepsilon_{4}}{\omega} \boldsymbol{Q}_{4} \cdot\left(\boldsymbol{Y}-\omega \boldsymbol{\theta}_{41}\right) .
\end{aligned}
$$

Here $\mathcal{J}$ is given in Eq. (12), $A\left(\boldsymbol{\Delta}_{\perp}\right)$ is the function $A(\boldsymbol{\Delta})$ at $\Delta_{\|}=0$, and the integration over $\boldsymbol{\Delta}_{\perp}$ is the integration over two-dimensional vector perpendicular to $z$-axis.

\section{COULOMB FIELD}

Let us consider the region $\min \left(\Delta_{0}, \Delta_{1}\right) \gg r_{s c r}^{-1}$, where $r_{s c r}$ is the screening radius and $\Delta_{1}=\sqrt{\left(\varepsilon_{2} \theta_{21}\right)^{2}+\left(m \omega / \varepsilon_{1}\right)^{2}}$. In this case we can neglect the effect of screening and use the Coulomb potential $V_{C}(r)=-\eta / r$ instead of the atomic potential $V(r)$. Then we have

$$
\begin{aligned}
& \chi(\rho)=-2 \eta \ln (2 L / \rho), \quad \mathcal{J}=\left(\frac{Q_{4}}{Q_{3}}\right)^{2 i \eta}-1, \\
& A_{C}(\Delta)=-\frac{4 \pi \eta(L \Delta)^{2 i \eta}}{\Delta^{2}} \Gamma(1-i \eta) \Gamma(2-i \eta) F\left(1-i \eta, i \eta, 2, \frac{\Delta_{\perp}^{2}}{\Delta^{2}}\right)
\end{aligned}
$$

where $F(a, b, c, x)$ is the hypergeometric function, $\Gamma(x)$ is the Euler $\Gamma$ function, $L \sim r_{s c r}$ and $A_{C}(\boldsymbol{\Delta})$ is $A(\boldsymbol{\Delta})$ in (10) for the Coulomb potential. At $\Delta_{\perp} \gg \Delta_{\|}$the function $A_{C}(\boldsymbol{\Delta})$ coincides with $A_{a s}(\boldsymbol{\Delta})$, Eq. (11).

In the Coulomb field the amplitudes $T_{\perp}^{(0)}$ and $T_{\|}^{(0)}$ are given by Eq. (14) with the replacement $A\left(\boldsymbol{\Delta}_{0}\right) \rightarrow A_{C}\left(\boldsymbol{\Delta}_{0}\right)$. To derive the amplitudes $T_{\perp}^{(1)}$ and $T_{\|}^{(1)}$ from Eq. (16), we integrate over the variables $\boldsymbol{Q}_{3}+\boldsymbol{Q}_{4}$ and $z$ in the same way as it was done in the case of the photoproduction cross section (see, e.g., [25]). The integration over $\boldsymbol{Q}_{34}=\boldsymbol{Q}_{3}-\boldsymbol{Q}_{4}$ is performed with the use of the Feynman parametrization. As a result we obtain

$$
\begin{aligned}
& T_{\perp}^{(1)}=\frac{8 i \eta \varepsilon_{1}}{\omega}|\Gamma(1-i \eta)|^{2} \int \frac{d \boldsymbol{\Delta}_{\perp} A_{a s}\left(\boldsymbol{\Delta}_{\perp}\right)}{Q^{2} M^{2}\left(m^{2} \omega^{2}+\varepsilon_{1}^{2} Y^{2}\right)}\left(\frac{\xi_{2}}{\xi_{1}}\right)^{i \eta} \mathcal{M} \\
& \mathcal{M}=-\frac{\delta_{\mu_{1} \mu_{2}} \delta_{\mu_{3} \bar{\mu}_{4}}}{\omega}\left[\varepsilon_{1}\left(\varepsilon_{3} \delta_{\mu_{1} \mu_{3}}-\varepsilon_{4} \delta_{\mu_{1} \mu_{4}}\right)\left(\boldsymbol{s}_{\mu_{1}}^{*} \cdot \boldsymbol{Y}\right)\left(\boldsymbol{s}_{\mu_{1}} \cdot \boldsymbol{I}_{1}\right)\right. \\
& \left.+\varepsilon_{2}\left(\varepsilon_{3} \delta_{\mu_{1} \bar{\mu}_{3}}-\varepsilon_{4} \delta_{\mu_{1} \bar{\mu}_{4}}\right)\left(\boldsymbol{s}_{\mu_{1}} \cdot \boldsymbol{Y}\right)\left(\boldsymbol{s}_{\mu_{1}}^{*} \cdot \boldsymbol{I}_{1}\right)\right]+\delta_{\mu_{1} \bar{\mu}_{2}} \delta_{\mu_{3} \bar{\mu}_{4}} \frac{m \omega \mu_{1}}{\sqrt{2} \varepsilon_{1}}\left(\varepsilon_{3} \delta_{\mu_{1} \mu_{3}}-\varepsilon_{4} \delta_{\mu_{1} \mu_{4}}\right)\left(\boldsymbol{s}_{\mu_{1}} \cdot \boldsymbol{I}_{1}\right) \\
& +\delta_{\mu_{1} \mu_{2}} \delta_{\mu_{3} \mu_{4}} \frac{m \mu_{3}}{\sqrt{2}}\left(\varepsilon_{1} \delta_{\mu_{1} \mu_{3}}+\varepsilon_{2} \delta_{\mu_{1} \bar{\mu}_{3}}\right)\left(\boldsymbol{s}_{\mu_{3}}^{*} \cdot \boldsymbol{Y}\right) I_{0}-\frac{m^{2} \omega^{2}}{2 \varepsilon_{1}} \delta_{\mu_{1} \bar{\mu}_{2}} \delta_{\mu_{3} \mu_{4}} \delta_{\mu_{1} \mu_{3}} I_{0} \\
& T_{\|}^{(1)}=-\frac{8 i \eta \varepsilon_{3} \varepsilon_{4}}{\omega^{3}}|\Gamma(1-i \eta)|^{2} \int \frac{d \boldsymbol{\Delta}_{\perp} A_{a s}\left(\boldsymbol{\Delta}_{\perp}\right)}{Q^{2} M^{2}}\left(\frac{\xi_{2}}{\xi_{1}}\right)^{i \eta} I_{0} \delta_{\mu_{1} \mu_{2}} \delta_{\mu_{3} \bar{\mu}_{4}}
\end{aligned}
$$


where the function $A_{a s}\left(\boldsymbol{\Delta}_{\perp}\right)$ is given in Eq. (11) and the following notations are used

$$
\begin{aligned}
& M^{2}=m^{2}\left(1+\frac{\varepsilon_{3} \varepsilon_{4}}{\varepsilon_{1} \varepsilon_{2}}\right)+\frac{\varepsilon_{1} \varepsilon_{3} \varepsilon_{4}}{\varepsilon_{2} \omega^{2}} Y^{2}, \quad \boldsymbol{Y}=\boldsymbol{\Delta}_{\perp}-\varepsilon_{2} \boldsymbol{\theta}_{21}, \quad \boldsymbol{\zeta}=\frac{\varepsilon_{3} \varepsilon_{4}}{\omega} \boldsymbol{\theta}_{34} \\
& \boldsymbol{Q}=\boldsymbol{\Delta}_{\perp}-\boldsymbol{\Delta}_{0}, \quad \boldsymbol{q}_{1}=\frac{\varepsilon_{3}}{\omega} \boldsymbol{Q}_{\perp}-\boldsymbol{\zeta}, \quad \boldsymbol{q}_{2}=\frac{\varepsilon_{4}}{\omega} \boldsymbol{Q}_{\perp}+\boldsymbol{\zeta} \\
& I_{0}=\left(\xi_{1}-\xi_{2}\right) F(x)+\left(\xi_{1}+\xi_{2}-1\right)(1-x) \frac{F^{\prime}(x)}{i \eta} \\
& \boldsymbol{I}_{1}=\left(\xi_{1} \boldsymbol{q}_{1}+\xi_{2} \boldsymbol{q}_{2}\right) F(x)+\left(\xi_{1} \boldsymbol{q}_{1}-\xi_{2} \boldsymbol{q}_{2}\right)(1-x) \frac{F^{\prime}(x)}{i \eta} \\
& \xi_{1}=\frac{M^{2}}{M^{2}+q_{1}^{2}}, \quad \xi_{2}=\frac{M^{2}}{M^{2}+q_{2}^{2}}, \quad x=1-\frac{Q_{\perp}^{2} \xi_{1} \xi_{2}}{M^{2}} \\
& F(x)=F(i \eta,-i \eta, 1, x), \quad F^{\prime}(x)=\frac{\partial}{\partial x} F(x) .
\end{aligned}
$$

Note that $Q_{\|}=-\Delta_{0 \|}$. In contrast to the term $T^{(0)}$, the Coulomb corrections to the term $T^{(1)}$ significantly modify the differential cross section of electroproduction not only at small $\Delta_{0}$ but also at $\Delta_{0} \sim m$.

\section{A. Born amplitude}

In the leading Born approximation, the terms $T_{B \perp}^{(0)}$ and $T_{B \|}^{(0)}$ are given by Eq. (14) with the replacement

$$
A\left(\boldsymbol{\Delta}_{0}\right) \rightarrow A_{B}\left(\boldsymbol{\Delta}_{0}\right)=-4 \pi \eta / \Delta_{0}^{2}
$$

To derive the terms $T_{B \perp}^{(1)}$ and $T_{B \|}^{(1)}$ from Eq. (18), we use the relation

$$
\lim _{\eta \rightarrow 0} \eta \int d \boldsymbol{\Delta}_{\perp} \Delta_{\perp}^{2 i \eta-2} G\left(\boldsymbol{\Delta}_{\perp}\right)=-i \pi G(0),
$$

where $G\left(\boldsymbol{\Delta}_{\perp}\right)$ is some function. Then we obtain

$$
\begin{aligned}
& T_{B \perp}^{(1)}=\frac{8 \pi \varepsilon_{1} \varepsilon_{2} A_{B}\left(\boldsymbol{\Delta}_{0}\right)}{\omega^{2} M_{B}^{2}\left(m^{2} \omega^{2}+\varepsilon_{1}^{2} \varepsilon_{2}^{2} \theta_{21}^{2}\right)} \mathcal{M}_{B}, \quad T_{B \|}^{(1)}=-\frac{8 \pi \varepsilon_{3} \varepsilon_{4} A_{B}\left(\boldsymbol{\Delta}_{0}\right)}{\omega^{3} M_{B}^{2}} I_{B 0} \delta_{\mu_{1} \mu_{2}} \delta_{\mu_{3} \bar{\mu}_{4}} \\
& \mathcal{M}_{B}=\delta_{\mu_{1} \mu_{2}} \delta_{\mu_{3} \bar{\mu}_{4}}\left[\varepsilon_{1}\left(\varepsilon_{3} \delta_{\mu_{1} \mu_{3}}-\varepsilon_{4} \delta_{\mu_{1} \mu_{4}}\right)\left(\boldsymbol{s}_{\mu_{1}}^{*} \cdot \boldsymbol{\theta}_{21}\right)\left(\boldsymbol{s}_{\mu_{1}} \cdot \boldsymbol{I}_{B 1}\right)\right. \\
& \left.+\varepsilon_{2}\left(\varepsilon_{3} \delta_{\mu_{1} \bar{\mu}_{3}}-\varepsilon_{4} \delta_{\mu_{1} \bar{\mu}_{4}}\right)\left(\boldsymbol{s}_{\mu_{1}} \cdot \boldsymbol{\theta}_{21}\right)\left(\boldsymbol{s}_{\mu_{1}}^{*} \cdot \boldsymbol{I}_{B 1}\right)\right]+\delta_{\mu_{1} \bar{\mu}_{2}} \delta_{\mu_{3} \bar{\mu}_{4}} \frac{m \omega^{2} \mu_{1}}{\sqrt{2} \varepsilon_{1} \varepsilon_{2}}\left(\varepsilon_{3} \delta_{\mu_{1} \mu_{3}}-\varepsilon_{4} \delta_{\mu_{1} \mu_{4}}\right)\left(\boldsymbol{s}_{\mu_{1}} \cdot \boldsymbol{I}_{B 1}\right) \\
& -\delta_{\mu_{1} \mu_{2}} \delta_{\mu_{3} \mu_{4}} \frac{m \mu_{3} \omega}{\sqrt{2}}\left(\varepsilon_{1} \delta_{\mu_{1} \mu_{3}}+\varepsilon_{2} \delta_{\mu_{1} \bar{\mu}_{3}}\right)\left(\boldsymbol{s}_{\mu_{3}}^{*} \cdot \boldsymbol{\theta}_{21}\right) I_{B 0}-\frac{m^{2} \omega^{3}}{2 \varepsilon_{1} \varepsilon_{2}} \delta_{\mu_{1} \bar{\mu}_{2}} \delta_{\mu_{3} \mu_{4}} \delta_{\mu_{1} \mu_{3}} I_{B 0} \\
& M_{B}^{2}=m^{2}\left(1+\frac{\varepsilon_{3} \varepsilon_{4}}{\varepsilon_{1} \varepsilon_{2}}\right)+\frac{\varepsilon_{1} \varepsilon_{2} \varepsilon_{3} \varepsilon_{4}}{\omega^{2}} \boldsymbol{\theta}_{21}^{2} \\
& I_{B 0}=\xi_{B 1}-\xi_{B 2}, \quad \boldsymbol{I}_{B 1}=\xi_{B 1} \boldsymbol{q}_{B 1}+\xi_{B 2} \boldsymbol{q}_{B 2}
\end{aligned}
$$




$$
\begin{aligned}
& \xi_{B 1}=\frac{M_{B}^{2}}{M_{B}^{2}+q_{B 1}^{2}}, \quad \xi_{B 2}=\frac{M_{B}^{2}}{M_{B}^{2}+q_{B 2}^{2}}, \quad \boldsymbol{\zeta}=\frac{\varepsilon_{3} \varepsilon_{4}}{\omega} \boldsymbol{\theta}_{34}, \\
& \boldsymbol{q}_{B 1}=-\frac{\varepsilon_{3}}{\omega} \boldsymbol{\Delta}_{0 \perp}-\boldsymbol{\zeta}, \quad \boldsymbol{q}_{B 2}=-\frac{\varepsilon_{4}}{\omega} \boldsymbol{\Delta}_{0 \perp}+\boldsymbol{\zeta} .
\end{aligned}
$$

The region, which gives the leading logarithmic contribution to the Born cross section, is

$$
m \ll \omega \ll \varepsilon_{1}, \quad \frac{m^{2}}{\omega} \ll \Delta_{0 \perp} \ll m, \quad \frac{m \omega}{\varepsilon_{1}} \ll \varepsilon_{1} \theta_{21} \ll m, \quad \zeta \sim m .
$$

In this region

$$
\begin{aligned}
& T_{B \perp}^{(1)}=\frac{32 \pi^{2} \eta \varepsilon_{1} \delta_{\mu_{1} \mu_{2}}}{\omega^{2}\left(m^{2}+\zeta^{2}\right) \Delta_{1 \perp}^{2} \Delta_{0 \perp}^{2}} \mathcal{M}_{a s}, \\
& \mathcal{M}_{a s}=\delta_{\mu_{3} \bar{\mu}_{4}}\left[\varepsilon_{3}\left(\boldsymbol{s}_{\mu_{3}}^{*} \cdot \boldsymbol{\theta}_{21}\right)\left(\boldsymbol{s}_{\mu_{3}} \cdot \boldsymbol{X}_{0}\right)-\varepsilon_{4}\left(\boldsymbol{s}_{\mu_{4}}^{*} \cdot \boldsymbol{\theta}_{21}\right)\left(\boldsymbol{s}_{\mu_{4}} \cdot \boldsymbol{X}_{0}\right)\right] \\
& -\mu_{3} \sqrt{2} m \omega \delta_{\mu_{3} \mu_{4}}\left(\boldsymbol{s}_{\mu_{3}}^{*} \cdot \boldsymbol{\theta}_{21}\right) \frac{\left(\boldsymbol{\zeta} \cdot \boldsymbol{\Delta}_{0 \perp}\right)}{m^{2}+\zeta^{2}} \\
& \boldsymbol{X}_{0}=\boldsymbol{\Delta}_{0 \perp}-\frac{2 \boldsymbol{\zeta}\left(\boldsymbol{\zeta} \cdot \boldsymbol{\Delta}_{0 \perp}\right)}{m^{2}+\zeta^{2}}
\end{aligned}
$$

and the terms $T_{B \perp}^{(0)}, T_{B \|}^{(0)}$, and $T_{B \|}^{(1)}$ are suppressed compared with $T_{B \perp}^{(1)}$. Substituting the matrix element (23) in (11) and performing the integration over the region (22) with the logarithmic accuracy, we obtain the well-known result [4, 6] for the leading logarithmic contribution to the total cross section of high-energy electroproduction

$$
\sigma=\frac{28 \eta^{2} \alpha^{2}}{27 \pi m^{2}} \ln ^{3} \frac{\varepsilon_{1}}{m}
$$

\section{B. Asymptotic forms of the exact in $\eta$ amplitudes in the Coulomb field.}

In the region (22) we have $|T|=\left|T_{B}\right|$, where $T$ is the exact in $\eta$ asymptotic result and $T_{B}$ is given by (23). For large $p_{\perp}$ of all outgoing particles, $\varepsilon_{2} \theta_{21} \gg m, \varepsilon_{3} \theta_{31} \gg m$, and $\varepsilon_{4} \theta_{41} \gg m$, but $\Delta_{0} \lesssim m$ we have

$$
\begin{aligned}
& T_{\perp}^{(0)}=\frac{8 \pi A_{C}\left(\boldsymbol{\Delta}_{0}\right)}{\varepsilon_{1} \varepsilon_{2} \varepsilon_{3} \varepsilon_{4} \omega c_{1} \theta_{34}^{2}} \delta_{\mu_{1} \mu_{2}} \delta_{\mu_{3} \bar{\mu}_{4}}\left[\varepsilon_{3}\left(\boldsymbol{s}_{\mu_{3}}^{*} \cdot \boldsymbol{X}_{1}\right)\left(\boldsymbol{s}_{\mu_{3}} \cdot \boldsymbol{\theta}_{34}\right)\left(\varepsilon_{1} \delta_{\mu_{1} \mu_{3}}+\varepsilon_{2} \delta_{\mu_{1} \mu_{4}}\right)\right. \\
& \left.-\varepsilon_{4}\left(\boldsymbol{s}_{\mu_{4}}^{*} \cdot \boldsymbol{X}_{1}\right)\left(\boldsymbol{s}_{\mu_{4}} \cdot \boldsymbol{\theta}_{34}\right)\left(\varepsilon_{1} \delta_{\mu_{1} \mu_{4}}+\varepsilon_{2} \delta_{\mu_{1} \mu_{3}}\right)\right] \\
& T_{\|}^{(0)}=\frac{16 \pi A_{C}\left(\boldsymbol{\Delta}_{0}\right)}{\omega^{2} c_{1}^{2}} \delta_{\mu_{1} \mu_{2}} \delta_{\mu_{3} \bar{\mu}_{4}}\left(\boldsymbol{\theta}_{21} \cdot \boldsymbol{\Delta}_{0 \perp}\right), \\
& c_{1}=\varepsilon_{2} \theta_{21}^{2}+\varepsilon_{3} \theta_{31}^{2}+\varepsilon_{4} \theta_{41}^{2}, \quad \boldsymbol{X}_{1}=\boldsymbol{\Delta}_{0 \perp}-\frac{2 \varepsilon_{1} \varepsilon_{2}}{\omega c_{1}}\left(\boldsymbol{\theta}_{21} \cdot \boldsymbol{\Delta}_{0 \perp}\right) \boldsymbol{\theta}_{21} .
\end{aligned}
$$


In this region the terms $T_{\perp}^{(1)}$ and $T_{\|}^{(1)}$ read

$$
\begin{aligned}
& T_{\perp}^{(1)}=-\frac{8 \pi A_{C}\left(\boldsymbol{\Delta}_{0}\right)}{\varepsilon_{1} \varepsilon_{2} \varepsilon_{3} \varepsilon_{4} c_{2} \theta_{21}^{2}} \delta_{\mu_{1} \mu_{2}} \delta_{\mu_{3} \bar{\mu}_{4}}\left[\varepsilon_{1}\left(\varepsilon_{3} \delta_{\mu_{1} \mu_{3}}-\varepsilon_{4} \delta_{\mu_{1} \mu_{4}}\right)\left(\boldsymbol{s}_{\mu_{1}}^{*} \cdot \boldsymbol{\theta}_{21}\right)\left(\boldsymbol{s}_{\mu_{1}} \cdot \boldsymbol{X}_{2}\right)\right. \\
& \left.+\varepsilon_{2}\left(\varepsilon_{3} \delta_{\mu_{1} \bar{\mu}_{3}}-\varepsilon_{4} \delta_{\mu_{1} \bar{\mu}_{4}}\right)\left(\boldsymbol{s}_{\mu_{1}} \cdot \boldsymbol{\theta}_{21}\right)\left(\boldsymbol{s}_{\mu_{1}}^{*} \cdot \boldsymbol{X}_{2}\right)\right] \\
& T_{\|}^{(1)}=\frac{16 \pi A_{C}\left(\boldsymbol{\Delta}_{0}\right)}{c_{2}^{2}} \delta_{\mu_{1} \mu_{2}} \delta_{\mu_{3} \bar{\mu}_{4}}\left(\boldsymbol{\theta}_{34} \cdot \boldsymbol{\Delta}_{0 \perp}\right), \\
& c_{2}=\varepsilon_{1} \varepsilon_{2} \theta_{21}^{2}+\varepsilon_{3} \varepsilon_{4} \theta_{34}^{2}, \quad \boldsymbol{X}_{2}=\boldsymbol{\Delta}_{0 \perp}-\frac{2 \varepsilon_{3} \varepsilon_{4}}{c_{2}}\left(\boldsymbol{\theta}_{34} \cdot \boldsymbol{\Delta}_{0 \perp}\right) \boldsymbol{\theta}_{34} .
\end{aligned}
$$

To derive this formula, we use the relation

$$
i \eta \int d \boldsymbol{\Delta}_{\perp} A_{a s}\left(\boldsymbol{\Delta}_{\perp}\right) \frac{\left(\boldsymbol{\Delta}_{\perp}-\boldsymbol{q}_{\perp}\right)}{\left(\boldsymbol{\Delta}_{\perp}-\boldsymbol{q}\right)^{2}}=-\pi \boldsymbol{q}_{\perp} A_{C}(\boldsymbol{q}),
$$

which is valid for any three-dimensional vector $\boldsymbol{q}=q_{\|} \boldsymbol{\nu}+\boldsymbol{q}_{\perp}$, where $\boldsymbol{\nu} \cdot \boldsymbol{q}_{\perp}=0$ and $\boldsymbol{\nu} \cdot \boldsymbol{\Delta}_{\perp}=0$.

In the region $\omega \ll \varepsilon_{1}$ and $\varepsilon_{2} \theta_{21} \ll \min \left(m, \Delta_{0}\right)$, which provides the applicability of the Weizsäcker-Williams approximation [7], the leading contribution to the amplitude of electroproduction is

$$
\begin{aligned}
& T_{\perp}^{(1)}=\frac{8 \pi A_{C}\left(\boldsymbol{\Delta}_{1}\right)}{\omega^{2} \Delta_{0}^{2} m^{2}}|\Gamma(1-i \eta)|^{2}\left(\frac{\xi_{W 2}}{\xi_{W 1}}\right)^{i \eta}\left(\boldsymbol{\Delta}_{1 \perp} \cdot \boldsymbol{T}_{W}\right) \delta_{\mu_{1} \mu_{2}}, \\
& \boldsymbol{T}_{W}=\delta_{\mu_{3} \bar{\mu}_{4}}\left[\left(\varepsilon_{3}\left(\boldsymbol{s}_{\mu_{3}} \cdot \boldsymbol{I}_{W 1}\right) \boldsymbol{s}_{\mu_{3}}^{*}-\varepsilon_{4}\left(\boldsymbol{s}_{\mu_{4}} \cdot \boldsymbol{I}_{W 1}\right) \boldsymbol{s}_{\mu_{4}}^{*}\right]-\delta_{\mu_{3} \mu_{4}} \frac{m \omega \mu_{3}}{\sqrt{2}} I_{W 0} \boldsymbol{s}_{\mu_{3}}^{*},\right. \\
& \boldsymbol{q}_{W 1}=-\frac{\varepsilon_{3}}{\omega} \boldsymbol{\Delta}_{0 \perp}-\boldsymbol{\zeta}, \quad \boldsymbol{q}_{W 2}=-\frac{\varepsilon_{4}}{\omega} \boldsymbol{\Delta}_{0 \perp}+\boldsymbol{\zeta}, \quad \boldsymbol{\zeta}=\frac{\varepsilon_{3} \varepsilon_{4}}{\omega} \boldsymbol{\theta}_{34}, \\
& I_{W 0}=\left(\xi_{W 1}-\xi_{W 2}\right) F\left(x_{W}\right)+\left(\xi_{W 1}+\xi_{W 2}-1\right)\left(1-x_{W}\right) \frac{F^{\prime}\left(x_{W}\right)}{i \eta}, \\
& \boldsymbol{I}_{W 1}=\left(\xi_{W 1} \boldsymbol{q}_{W 1}+\xi_{W 2} \boldsymbol{q}_{W 2}\right) F\left(x_{W}\right)+\left(\xi_{W 1} \boldsymbol{q}_{W 1}-\xi_{W 2} \boldsymbol{q}_{W 2}\right)\left(1-x_{W}\right) \frac{F^{\prime}\left(x_{W}\right)}{i \eta}, \\
& \xi_{W 1}=\frac{m^{2}}{m^{2}+q_{W 1}^{2}}, \quad \xi_{W 2}=\frac{m^{2}}{m^{2}+q_{W 2}^{2}}, \quad x_{W}=1-\frac{\Delta_{0 \perp}^{2} \xi_{W 1} \xi_{2 W}}{m^{2}},
\end{aligned}
$$

where $\boldsymbol{\Delta}_{1 \perp}=\varepsilon_{2} \boldsymbol{\theta}_{21}, \Delta_{1 \|}=m \omega / \varepsilon_{1}$, and the functions $F(x)$ and $F^{\prime}(x)$ are defined in (19). Note that the amplitude $T_{\text {real }}$ of $e^{+} e^{-}$photoproduction by a real photon with the polarization vector $\boldsymbol{e}$ is [24]

$$
T_{\text {real }}=\frac{8 \pi \eta}{\omega \Delta_{0}^{2} m^{2}}|\Gamma(1-i \eta)|^{2}\left(\frac{\xi_{W 2}}{\xi_{W 1}}\right)^{i \eta}\left(\boldsymbol{e} \cdot \boldsymbol{T}_{W}\right),
$$

where the function $\boldsymbol{T}_{W}$ is the same as in Eq. (28).

In the leading logarithmic approximation, the Coulomb corrections to the cross section in the Coulomb field, proportional to $\ln ^{2}\left(\varepsilon_{1} / m\right)$, are originated from three regions:

$$
\text { 1. } \frac{m^{2}}{\omega} \sim \Delta_{0 \perp}, \quad \max \left\{\frac{m \omega}{\varepsilon_{1}}, \Delta_{0 \perp}\right\} \ll \Delta_{1 \perp} \ll m,
$$




$$
\begin{aligned}
& \text { 2. } \max \left\{\Delta_{1 \perp}, \frac{m^{2}}{\omega}\right\} \ll \Delta_{0 \perp} \ll m, \quad \frac{m \omega}{\varepsilon_{1}} \sim \Delta_{1 \perp}, \\
& \text { 3. } \Delta_{0} \sim m, \quad \frac{m \omega}{\varepsilon_{1}} \ll \Delta_{1 \perp} \ll m,
\end{aligned}
$$

where in all regions $m \ll \omega \ll \varepsilon_{1}$ and $\zeta \sim m$. Using Eq. (18), we find the amplitude $T_{\perp}^{(1)}$ in the first region in (30):

$$
T_{\perp}^{(1)}=-\frac{8 \pi \varepsilon_{1} A_{C}\left(\Delta_{0}\right) \delta_{\mu_{1} \mu_{2}}}{\omega^{2}\left(m^{2}+\zeta^{2}\right) \Delta_{1 \perp}^{2}} \mathcal{M}_{a s}
$$

where $\mathcal{M}_{a s}$ is given in Eq. (23) $)$. In the second region in (30), the amplitude $T_{\perp}^{(1)}$ has the form

$$
T_{\perp}^{(1)}=-\frac{8 \pi \varepsilon_{1} A_{C}\left(\Delta_{1}\right) \delta_{\mu_{1} \mu_{2}}}{\omega^{2}\left(m^{2}+\zeta^{2}\right) \Delta_{0 \perp}^{2}} \mathcal{M}_{a s}
$$

To derive Eqs.(31) and (32), we use the relation (27). The expression for $T_{\perp}^{(1)}$ in the third region in (30) is given by Eq. (28) with the replacement $A_{C}\left(\Delta_{1}\right) \rightarrow A_{a s}\left(\Delta_{1}\right)$. In all regions in (301), the terms $T_{\perp}^{(0)}, T_{\|}^{(0)}$, and $T_{\|}^{(1)}$ are suppressed compared with $T_{\perp}^{(1)}$.

Performing calculations with the logarithmic accuracy, we find that the contributions of the first and second regions in (30) are equal to each other and two times smaller than the contribution of the third region, so that the total result reads

$$
\sigma_{C}=-\frac{56 \eta^{2} \alpha^{2}}{9 \pi m^{2}} f(\eta) \ln ^{2} \frac{\varepsilon_{1}}{m}, \quad f(\eta)=\operatorname{Re}[\psi(1+i \eta)-\psi(1)],
$$

where $\psi(x)=d \Gamma(x) / d x$. However the origin of these contributions are different. The contribution of the third region can be easily obtained within Weizsäcker-Williams approximation. It corresponds to the Coulomb corrections to the cross section of electroproduction in the Coulomb field by a relativistic particle noninteracting with this field [27, 28]. Therefore, the Coulomb corrections coming from the third region are given by the momentum transfer $\Delta_{0} \sim m$. Using the language of exchanges by the Coulomb quanta with the nucleus, we can say that the contributions to the Coulomb corrections of the first and second regions in (30) correspond to the case when all particles interact with the Coulomb center. In this case, to derive the Coulomb corrections to the total cross section from Eqs. (31) and (32), it is necessary to use the relation [23, 28]

$$
\int d \boldsymbol{\Delta}_{\perp} \Delta_{\perp}^{2}\left[\left|A_{C}(\boldsymbol{\Delta})\right|^{2}-\left|A_{B}(\boldsymbol{\Delta})\right|^{2}\right]=-32 \pi^{3} \eta^{2} f(\eta)
$$

Therefore, the Coulomb corrections coming from the first and second regions are given by the small momentum transfer $\Delta_{0}, \Delta_{1} \ll m$. 


\section{EFFECT OF SCREENING}

The effect of screening is important if $\Delta_{0} \lesssim r_{s c r}^{-1}$ or $\Delta_{1} \lesssim r_{s c r}^{-1}$. In this case the main contribution to the integrals in (16) is given by $\Delta_{\perp} \sim \min \left(\Delta_{0}, \Delta_{1}\right)$. The effect of screening in the amplitude $J_{\lambda}^{(1)}$ of photoproduction by a virtual photon (12) can be taken into account similar to the case of photoproduction by a real photon. Namely, one should multiply $J_{\lambda}^{(1)}$ in the Coulomb field by the atomic form factor $F\left(Q^{2}\right)$, where $\boldsymbol{Q}=\boldsymbol{p}_{3}+\boldsymbol{p}_{4}-\boldsymbol{k}$. This recipe is valid, because at $Q \ll m$ screening affect only the leading in $\eta$ term of $J_{\lambda}^{(1)}$, while the Coulomb corrections to $J_{\lambda}^{(1)}$ are originated from the region $Q \sim m$ where $F\left(Q^{2}\right)=1$. Screening is taken into account in the matrix element $j_{\lambda}$ of virtual photon bremsstrahlung (10) via the function $A(\boldsymbol{\Delta})$ in the atomic field, Eq. (10) . Thus, the terms $T_{\perp}^{(0)}$ and $T_{\|}^{(0)}$ in the amplitude of electroproduction in the atomic field are given directly by Eq. (14). Then, multiplying the integrands in Eq. (18) for the Coulomb field by the atomic form factor $F\left(Q^{2}\right)$ and making the replacement $A_{a s}\left(\boldsymbol{\Delta}_{\perp}\right) \rightarrow A\left(\boldsymbol{\Delta}_{\perp}\right)$, we obtain the terms $T_{\perp}^{(1)}$ and $T_{\|}^{(1)}$ in the atomic field. This result will be valid for any values of $\Delta_{0}$ and $\Delta_{1}$.

Let us now discuss the impact of screening on the total cross section of electroproduction. To obtain the result in the leading logarithmic approximation, one should consider the region (cf. (22) )

$$
m \ll \omega \ll \varepsilon_{1}, \quad \max \left\{\frac{m^{2}}{\omega}, r_{s c r}^{-1}\right\} \ll \Delta_{0 \perp} \ll m, \quad \frac{m \omega}{\varepsilon_{1}} \ll \varepsilon_{1} \theta_{21} \ll m, \quad \frac{\varepsilon_{3} \varepsilon_{4}}{\omega} \theta_{34} \sim m,
$$

where the leading term of the cross section coincides with the Born result. Under the conditions (35), the main contribution to the amplitude $T_{B}$ is given by the terms $T_{B \perp}^{(1)}$ (23), while the terms $T_{B \perp}^{(0)}, T_{B \|}^{(0)}$, and $T_{B \|}^{(1)}$ are suppressed. Performing the integration with the logarithmic accuracy, we obtain for $\varepsilon_{1}>m^{2} r_{s c r} \sim m Z^{-1 / 3} / \alpha$

$$
\sigma=\frac{28 \eta^{2} \alpha^{2}}{27 \pi m^{2}}\left[\ln ^{3}\left(m r_{s c r}\right)+3 \ln \frac{\varepsilon_{1}}{m} \ln \left(m r_{s c r}\right) \ln \frac{\varepsilon_{1}}{m^{2} r_{s c r}}\right]
$$

This result coincides with that obtained in Ref. [10]. For $m \ll \varepsilon_{1}<m^{2} r_{\text {scr }}$ the total cross section in the leading logarithmic approximation is independent of $r_{\text {scr }}$ and coincides with Eq. (24). Note that the asymptotics (36) has good accuracy only at very high energy. To obtain the result with high accuracy, it is necessary to perform the integration of our results beyond the leading logarithmic approximation.

In the leading logarithmic approximation, the Coulomb corrections to the total cross 
section of electroproduction in the atomic field are originated from three regions (cf. (30)):

$$
\begin{aligned}
& \text { 1. } \max \left\{\frac{m^{2}}{\omega}, r_{s c r}^{-1}\right\} \sim \Delta_{0 \perp}, \quad \max \left\{\frac{m \omega}{\varepsilon_{1}}, \Delta_{0 \perp}\right\} \ll \Delta_{1 \perp} \ll m, \\
& \text { 2. } \max \left\{\Delta_{1 \perp}, \frac{m^{2}}{\omega}\right\} \ll \Delta_{0 \perp} \ll m, \quad \max \left\{\frac{m \omega}{\varepsilon_{1}}, r_{s c r}^{-1}\right\} \sim \Delta_{1 \perp}, \\
& \text { 3. } \Delta_{0} \sim m, \quad \frac{m \omega}{\varepsilon_{1}} \ll \Delta_{1 \perp} \ll m,
\end{aligned}
$$

where in all regions $m \ll \omega \ll \varepsilon_{1}$ and $\zeta=\varepsilon_{3} \varepsilon_{4} \theta_{34} / \omega \sim m$.

To obtain the amplitude $T_{\perp}^{(1)}$ in the first region in (37), we use the following transformation

$$
\begin{aligned}
& i \eta \int d \boldsymbol{\Delta}_{\perp} A\left(\boldsymbol{\Delta}_{\perp}\right) F\left(\left(\boldsymbol{q}-\boldsymbol{\Delta}_{\perp}\right)^{2}\right) \frac{\left(\boldsymbol{q}_{\perp}-\boldsymbol{\Delta}_{\perp}\right)}{\left(\boldsymbol{q}-\boldsymbol{\Delta}_{\perp}\right)^{2}} \\
& =-\frac{1}{4 \pi} \int d \boldsymbol{\Delta}_{\perp} d \boldsymbol{r} A\left(\boldsymbol{\Delta}_{\perp}\right) \boldsymbol{\nabla}_{\perp} V(\boldsymbol{r}) e^{-i\left(\boldsymbol{q}-\boldsymbol{\Delta}_{\perp}\right) \boldsymbol{r}} \\
& =-i \pi \int d \boldsymbol{r} \boldsymbol{\nabla}_{\perp} \boldsymbol{V}(\boldsymbol{r}) \exp [-i \chi(\rho)-i \boldsymbol{q} \cdot \boldsymbol{r}]=\pi \boldsymbol{q}_{\perp} A(\boldsymbol{q}),
\end{aligned}
$$

where the function $A(\boldsymbol{\Delta})$ is given in Eq. (10). Then the amplitude $T_{\perp}^{(1)}$ in the first region in (37) is

$$
T_{\perp}^{(1)}=-\frac{8 \pi \varepsilon_{1} A\left(\boldsymbol{\Delta}_{0}\right) \delta_{\mu_{1} \mu_{2}}}{\omega^{2}\left(m^{2}+\zeta^{2}\right) \Delta_{1 \perp}^{2}} \mathcal{M}_{a s}
$$

where $\mathcal{M}_{a s}$ is given in Eq. (31). Similar to Eq. (39), we obtain the amplitude $T_{\perp}^{(1)}$ in the second region in (37):

$$
\begin{aligned}
& T_{\perp}^{(1)}=-\frac{8 \pi \varepsilon_{1} A_{1}\left(\boldsymbol{\Delta}_{1}\right) \delta_{\mu_{1} \mu_{2}}}{\omega^{2}\left(m^{2}+\zeta^{2}\right) \Delta_{0 \perp}^{2}} \mathcal{M}_{a s}, \\
& A_{1}(\boldsymbol{\Delta})=-\frac{i}{\Delta_{\perp}^{2}} \int d \boldsymbol{r} \exp [-i \boldsymbol{\Delta} \cdot \boldsymbol{r}-i \chi(\rho)] \boldsymbol{\Delta}_{\perp} \cdot \boldsymbol{\nabla}_{\perp} V_{C}(r) .
\end{aligned}
$$

The amplitude $T_{\perp}^{(1)}$ in the third region in (37) is independent of $r_{s c r}$ and coincides with the expression (28) with the replacement $A_{C}\left(\Delta_{1}\right) \rightarrow A_{a s}\left(\Delta_{1}\right)$, Eq. (111). In all regions in (37), the terms $T_{\perp}^{(0)}, T_{\|}^{(0)}$, and $T_{\|}^{(1)}$ are suppressed compared with $T_{\perp}^{(1)}$.

For the total cross section, the contributions of the first and second regions to the Coulomb corrections calculated within logarithmic accuracy are equal to each other,

$$
\delta \sigma_{C}^{(1)}=\delta \sigma_{C}^{(2)}=-\frac{56 \eta^{2} \alpha^{2}}{9 \pi m^{2}} f(\eta) \ln \left(m r_{c s r}\right) \ln \frac{\varepsilon_{1}}{m^{2} r_{c s r}}
$$

for $\varepsilon_{1}>m^{3} r_{s c r}^{2}$ and

$$
\delta \sigma_{C}^{(1)}=\delta \sigma_{C}^{(2)}=-\frac{14 \eta^{2} \alpha^{2}}{9 \pi m^{2}} f(\eta) \ln ^{2} \frac{\varepsilon_{1}}{m}
$$


for $m \ll \varepsilon_{1}<m^{3} r_{s c r}^{2}$. To derive Eqs. (41) and (42) we use the relations [23]

$$
\begin{aligned}
& \int d \boldsymbol{\Delta}_{0 \perp} \Delta_{0 \perp}^{2}\left[\left|A\left(\boldsymbol{\Delta}_{0}\right)\right|^{2}-\left|A_{B}\left(\boldsymbol{\Delta}_{0}\right)\right|^{2}\right]=-32 \pi^{3} \eta^{2} f(\eta), \\
& \int d \boldsymbol{\Delta}_{1 \perp} \Delta_{1 \perp}^{2}\left[\left|A_{1}\left(\boldsymbol{\Delta}_{1}\right)\right|^{2}-\left|A_{1 B}\left(\boldsymbol{\Delta}_{1}\right)\right|^{2}\right]=-32 \pi^{3} \eta^{2} f(\eta), \\
& A_{1 B}\left(\boldsymbol{\Delta}_{1}\right)=-4 \pi \eta / \Delta_{1}^{2},
\end{aligned}
$$

valid for any atomic potential $V(r)$. The contribution of the third region is independent of $r_{\text {scr }}$ and equals

$$
\delta \sigma_{C}^{(3)}=-\frac{28 \eta^{2} \alpha^{2}}{9 \pi m^{2}} f(\eta) \ln ^{2} \frac{\varepsilon_{1}}{m}
$$

for all $\varepsilon_{1} \gg m$. Thus, the total Coulomb corrections $\sigma_{C}=\delta \sigma_{C}^{(1)}+\delta \sigma_{C}^{(2)}+\delta \sigma_{C}^{(3)}$ are

$$
\sigma_{C}=-\frac{28 \eta^{2} \alpha^{2}}{9 \pi m^{2}} f(\eta)\left[\ln ^{2} \frac{\varepsilon_{1}}{m}+4 \ln \left(m r_{c s r}\right) \ln \frac{\varepsilon_{1}}{m^{2} r_{c s r}}\right]
$$

for $\varepsilon_{1}>m^{3} r_{s c r}^{2}$ and

$$
\sigma_{C}=-\frac{56 \eta^{2} \alpha^{2}}{9 \pi m^{2}} f(\eta) \ln ^{2} \frac{\varepsilon_{1}}{m}
$$

for $m \ll \varepsilon_{1}<m^{3} r_{s c r}^{2}$, see Eq. (33). Note that the contribution $\delta \sigma_{C}^{(1)}+\delta \sigma_{C}^{(2)}$, coming from small momentum transfers $\Delta_{0} \ll m$, approximately equals to $\delta \sigma_{C}^{(3)}$, coming from $\Delta_{0} \sim m$, up to very high energy $\varepsilon_{1}$.

\section{IMPACT OF THE COULOMB CORRECTIONS ON THE DIFFERENTIAL CROSS SECTION}

To compare the exact in $\eta$ differential cross section with the Born result, we introduce the dimensionless quantity $\mathrm{S}$,

$$
S=\sum_{\mu_{1} \mu_{2} \mu_{3} \mu_{4}}\left|\frac{\varepsilon_{1} m^{4} \mathcal{T}_{\mu_{1} \mu_{2} \mu_{3} \mu_{4}}}{\eta(2 \pi)^{2}}\right|^{2}
$$

which is the normalized differential cross section summed over polarizations of all particles. We consider the region $\Delta_{0 \|} \gg 1 / r_{s c r}$ and $\omega / \varepsilon_{1} \gg 1 /\left(m r_{s c r}\right)$, where it is not necessary to take screening into account. Besides, for $\Delta_{0 \perp} \gg \Delta_{0 \|}$ the function $S$ depends only on the ratios $\varepsilon_{i} / \varepsilon_{1}$, but not on the energy $\varepsilon_{1}$ itself. 


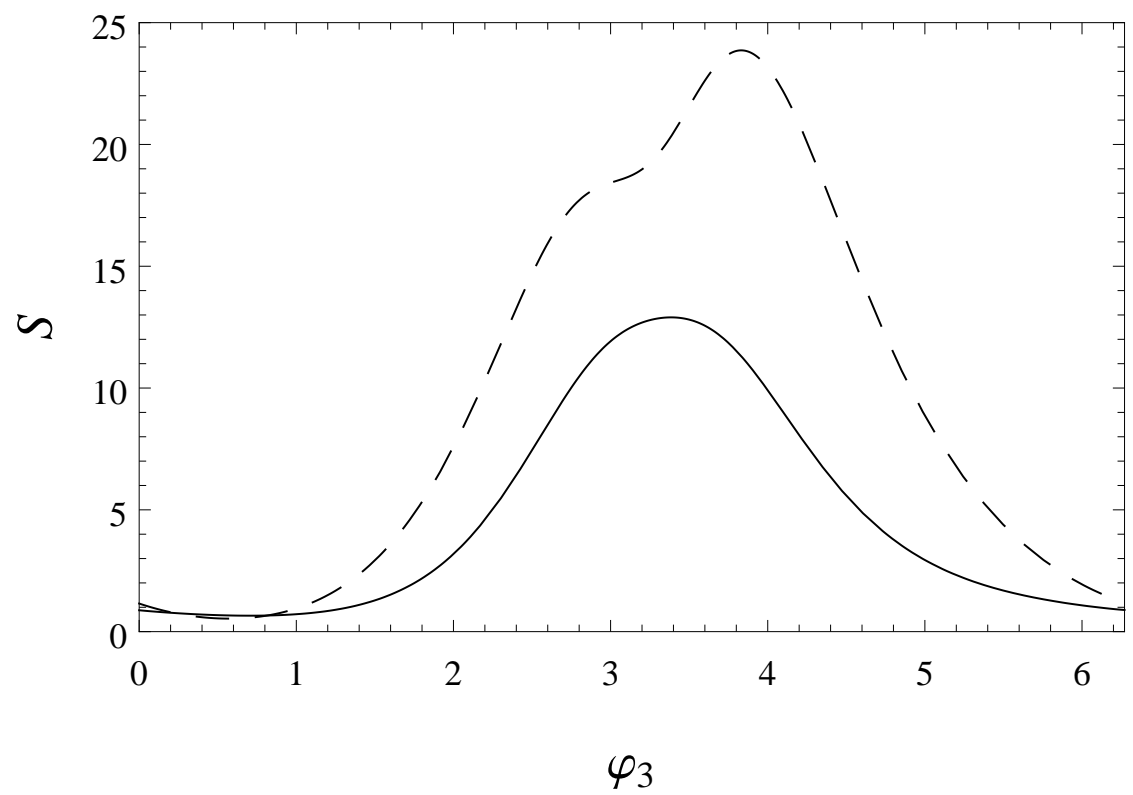

FIG. 2: The quantity $S$, see Eq. (47), as a function of the azimuth angle $\varphi_{3}$ for $\varepsilon_{2} / \varepsilon_{1}=0.28$, $\varepsilon_{3} / \varepsilon_{1}=0.42, \varepsilon_{4} / \varepsilon_{1}=0.3, p_{2 \perp}=0.3 m, p_{3 \perp}=0.5 m, p_{4 \perp}=1.2 m$, and $\varphi_{2}=\pi / 4 ;$ the Born result (dashed curve) and the exact in $\eta$ result for $\eta=0.6$ (solid curve).

We direct $z$-axis along $\boldsymbol{p}_{1}$ and $x$-axis along $\boldsymbol{p}_{4 \perp}$. In Fig. 2 we show the dependence of $S$ on $\varphi_{3}$ at some values of $\varepsilon_{i}, p_{i \perp}$, and $\varphi_{2}$, where $\varphi_{2}$ is the azimuth angle of $\boldsymbol{p}_{2 \perp}$ and $\varphi_{3}$ is the azimuth angle of $\boldsymbol{p}_{3 \perp}$.

In Fig. 3 the dependence of $S$ on $\delta_{4}=p_{4 \perp} / m$ is shown at some values of $\varepsilon_{i}$ and $\boldsymbol{p}_{i \perp}$. In the right picture in Fig. 3 the point $\delta_{4}=0.8$, where $S=0$, corresponds to the momentum transfer $\Delta_{0 \perp}=0$. It is seen from Figs. 2 and 3 that the Coulomb corrections significantly modify the differential cross section compared with the Born result.

The exact in $\eta$ differential cross section for the polarized incoming particle possesses the azimuth asymmetry $\mathcal{A}$,

$$
\mathcal{A}=\frac{S_{+}-S_{-}}{S_{+}+S_{-}}, \quad S_{ \pm}=\sum_{\mu_{2} \mu_{3} \mu_{4}}\left|\frac{\varepsilon_{1} m^{4} \mathcal{T}_{ \pm \mu_{2} \mu_{3} \mu_{4}}}{\eta(2 \pi)^{2}}\right|^{2} .
$$

In the Born approximation the asymmetry vanishes for any $\boldsymbol{p}_{i}$ due to the relation

$$
\mathcal{T}_{\mu_{1} \mu_{2} \mu_{3} \mu_{4}}^{B}=-\mu_{1} \mu_{2} \mu_{3} \mu_{4}\left(\mathcal{T}_{\bar{\mu}_{1} \bar{\mu}_{2} \bar{\mu}_{3} \bar{\mu}_{4}}^{B}\right)^{*}
$$

following from Eq. (21). However, this relation is not valid for the Coulomb corrections because the integrand in Eq. (18) is not a real quantity. The asymmetry $\mathcal{A}$ is shown in 

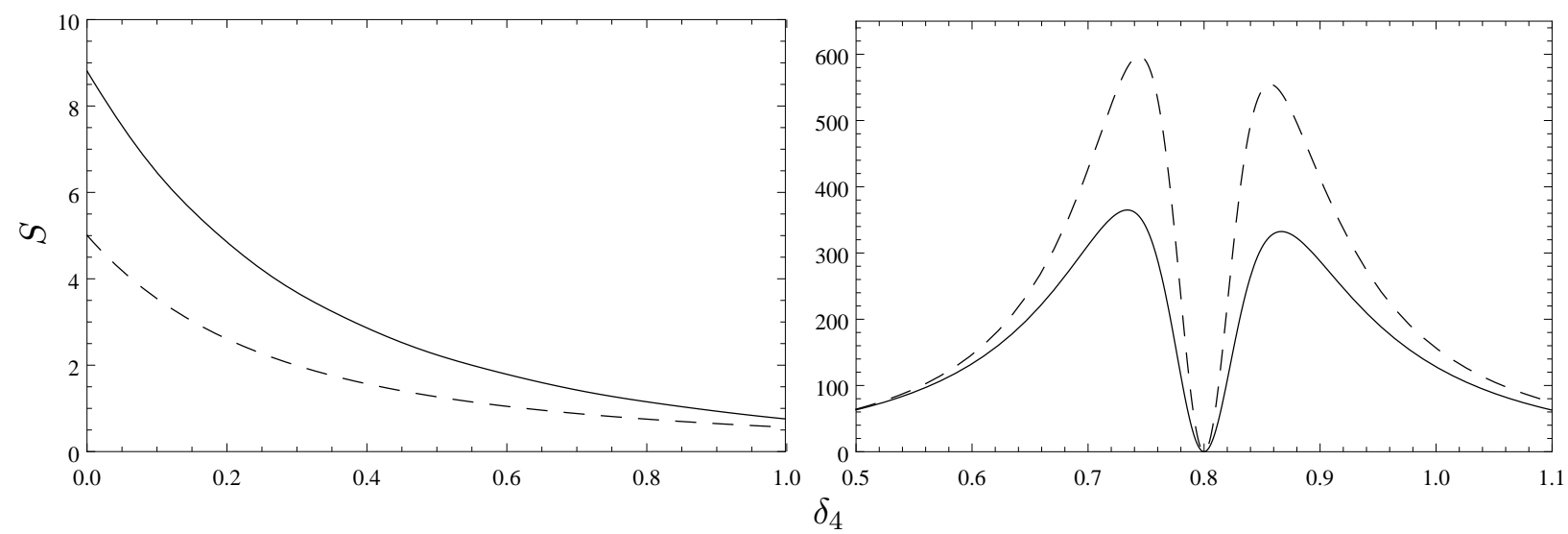

FIG. 3: The quantity $S$, see Eq. (47), as a function of $\delta_{4}=p_{4 \perp} / \mathrm{m}$ for $\varepsilon_{1}=100 m, \varepsilon_{2} / \varepsilon_{1}=0.28$, $\varepsilon_{3} / \varepsilon_{1}=0.42, \varepsilon_{4} / \varepsilon_{1}=0.3, p_{2 \perp}=0.3 m, p_{3 \perp}=0.5 m, \varphi_{2}=\varphi_{3}=0$ (left picture), and $\varphi_{2}=\varphi_{3}=\pi$ (right picture); Born result (dashed curve) and the exact in $\eta$ result for $\eta=0.6$ (solid curve).

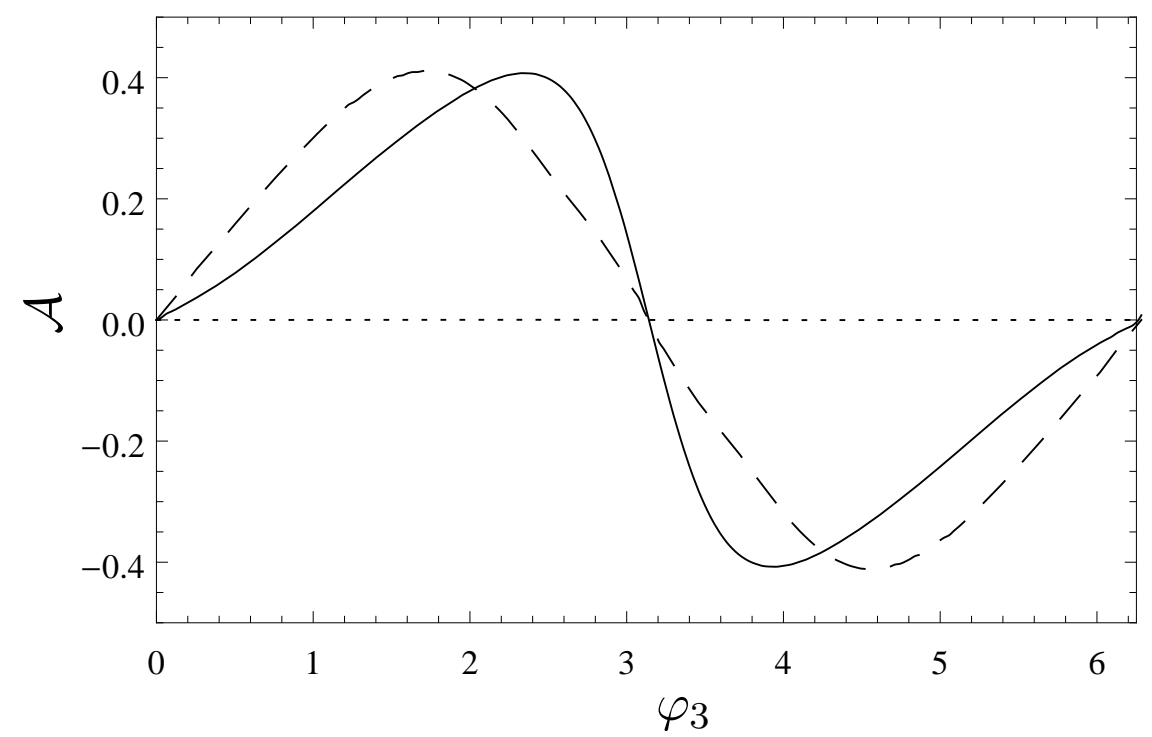

FIG. 4: The quantity $\mathcal{A}$, see Eq. (48), as a function of $\varphi_{3}$ for $\varepsilon_{2} / \varepsilon_{1}=0.28, \varepsilon_{3} / \varepsilon_{1}=0.42$, $\varepsilon_{4} / \varepsilon_{1}=0.3, p_{2 \perp}=0.3 m, p_{3 \perp}=0.5 m, p_{4 \perp}=1.2 m, \eta=0.6 ; \varphi_{2}=0$ (solid curve) and $\varphi_{2}=\pi$ (dashed curve). In the Born approximation $\mathcal{A}=0$ (dotted curve).

Fig. 4 as a function of $\varphi_{3}$ at some values of $\varepsilon_{i}, p_{i \perp}$, and $\varphi_{2}$. As it should be, the asymmetry vanishes when all momenta are in the same plane $\left(\varphi_{2}=0, \pi\right.$ and $\varphi_{3}=0, \pi$ in Fig. 4). It is seen that the asymmetry can reach tens of percent. 


\section{CONCLUSION}

Using the quasiclassical approximation, we have derived the exact in the parameter $\eta$ differential cross section of high-energy electroproduction in the atomic field. The helicity amplitudes of the process for the Coulomb field are given in Eqs. (14) and (18), and the modification of these formulas for the atomic field (the effect of screening) are pointed out in Sec. IV, The Coulomb corrections substantially modify the differential cross section compared with the Born result. For the polarized incoming electron, the Coulomb corrections lead to the azimuth asymmetry in the differential cross section, Eq. (48). The leading logarithmic contribution to the Coulomb corrections to the total cross section is given not only by moderate momentum transfers $\Delta_{0} \sim m$, but also by small momentum transfers $\Delta_{0} \ll m$. Emphasize that the latter contribution appears due to interaction of the incoming electron with the atomic field.

\section{Acknowledgement}

We are grateful to R.N. Lee for important discussions. This work has been supported by Russian Science Foundation (Project No. 14-50-00080). It has been also supported in part by RFBR (Grant No. 16-02-00103).

[1] A1 Collaboration (H. Merkel et al.) Phys.Rev.Lett. 106, 251802 (2011).

[2] APEX Collaboration (S. Abrahamyan et al.) Phys.Rev.Lett. 107, 191804 (2011).

[3] H. Bhabha, Proc. Cambridge Phil. Soc. 31, 394 (1935).

[4] H. Bhabha, Proc. Roy. Soc. (London) A152, 559 (1935).

[5] G. Racah, Nuovo Cimento 4, 66 (1936).

[6] G. Racah, Nuovo Cimento 4, 112 (1937).

[7] V. B. Berestetski, E. M. Lifshits, and L. P. Pitayevsky, Quantum Electrodynamics (Pergamon, Oxford, 1982).

[8] M. M. Block, D. T. King, and M. Wada, Phys. Rev. 96, 1627 (1954).

[9] T. Murota and A. Veda, Progr. Theoret. Phys. (Kyoto) 16, 497 (1956).

[10] T. Murota, A. Veda, and H. Tanaka, Progr. Theoret. Phys.(Kyoto) 16, 482 (1956). 
[11] E. G. Johnson Phys.Rev 140, 1005 (1965).

[12] S. Brodsky and S. Ting, Phys. Rev. 145, 1018 (1966).

[13] J. D. Bjorken and M. Chen, Phys. Rev. 154, 1335 (1967).

[14] R. Henry, Phys. Rev. 154, 1534 (1967).

[15] S. Homma, A . Itano, K . Nishikawa, and M. Hayashi, Proc. Phys. Soc. Japan 3, 1230 (1974).

[16] H. A. Bethe and L. C. Maximon, Phys. Rev. 93, 768 (1954).

[17] H. Davies, H. A. Bethe, and L. C. Maximon, Phys. Rev. 93, 788 (1954).

[18] H. A. Olsen, L. C. Maximon, and H. Wergeland, Phys. Rev. 106, 27 (1957).

[19] W. Furry, Phys. Rev. 46, 391 (1934).

[20] A. Sommerfeld, A. Maue, Ann. Phys. 22, 629 (1935).

[21] R. N. Lee, A. I. Milstein, V. M. Strakhovenko, Zh. Eksp. Teor. Fiz. 117, 75 (2000) [JETP 90, $66(2000)]$.

[22] P. A. Krachkov and A. I. Milstein, Phys. Rev. A 91, 032106 (2015).

[23] R. N. Lee, A. I. Milstein, V. M. Strakhovenko, and O. Ya. Schwarz, Zh. Eksp. Teor. Fiz. 127, 5 (2005) [JETP 100, 1 (2005)].

[24] R. N. Lee, A. I. Milstein, and V. M. Strakhovenko, Phys. Rev. A 85, 042104 (2012).

[25] P. A. Krachkov, R. N. Lee, and A. I. Milstein, Phys. Rev. A 90, 062112 (2014).

[26] P. A. Krachkov, R.N. Lee, and A. I. Milstein, Phys. Rev. A 91, 062109 (2015).

[27] D. Yu. Ivanov, E. A. Kuraev, A. Schiller, and V. G. Serbo, Phys. Lett. B 442, 453 (1998).

[28] R.N. Lee, A.I. Milstein, Phys. Rev. A 61, 032103 (2000). 